enough blood for HIV, creatinine, and quantitative rapid plasma reagin testing.

From May 2020 - January 2021, we recruited subjects for two studies of the Tasso device and HBPM preferences. First, we sent invitations to English-speaking clients enrolled in an online messaging platform and receiving PrEP through the Sexual Health Clinic (SHC) in Seattle, WA, asking them to view a Tasso device video and complete a survey. In a second study, we recruited SHC PrEP clients and persons with known/suspected syphilis or living with HIV. After brief instruction in clinic, clients used the Tasso device and completed a survey about their experience.

Of 509 clients offered participation online and 46 in clinic, $102(20 \%)$ and 46 (100\%), respectively, completed surveys. Most participants were cisgender men $(132,90 \%)$ of median age 34 years who identified as non-Hispanic White (78, $52.7 \%$ ). Thirty-seven (45\%) reported a history of syphilis. Given current COVID restrictions, 124 (89.2\%) participants were interested in self-collecting PrEP laboratory samples at home. Many felt home sampling kits would increase their likelihood of staying on PrEP $(61 / 135,45.2 \%)$. Of the 46 participants who self-collected blood specimens in clinic, 42 (93.3\%) felt the process was easy and 35/36 (97.2\%) PrEP-eligible participants felt they could similarly self-collect blood at home.

Self-collection of blood specimens using the Tasso device is feasible and acceptable to persons using PrEP. Future studies will assess how often individuals will use this device rather than come to the SHC for PrEP care and the impact of HBPM on PrEP retention.

\section{P114 A NEW METHOD FOR SELF-COLLECTION OF BLOOD SPECIMENS FOR HIV PRE-EXPOSURE PROPHYLAXIS (PREP) LABORATORY MONITORING}

${ }^{1,2} \mathrm{C}$ Cannon* ${ }^{*}{ }^{1,2} \mathrm{C}$ Malinski, ${ }^{1,2} \mathrm{M}$ Golden. ${ }^{1}$ University of Washington, Seattle, USA; ${ }^{2}$ Public Health-Seattle and King County, Seattle, USA

10.1136/sextrans-2021-sti.236

Sexually transmitted infection (STI) testing is an important component of PrEP care. Home-based PrEP monitoring (HBPM), consisting of STI sample self-collection and remote follow-up, could improve PrEP access and retention rates, particularly in the context of the COVID-19 epidemic. However, existing fingerstick methods for collecting blood often result in too little specimen for quantitative rapid plasma reagin (RPR) testing, limiting the ability to distinguish between old and new syphilis infections. We evaluated the Tasso OnDemand $^{\mathrm{TM}}$ device, designed to collect $300 \mu \mathrm{g}$ of capillary blood, for self-collecting blood specimens for PrEP monitoring, including quantitative syphilis testing.

From July 2020 - January 2021, we enrolled 46 clients who were either on PrEP, had known/suspected new syphilis infection or were living with HIV and seeking STI care at a sexual health clinic in Seattle, WA, USA. After brief instruction, clients used the Tasso device in the clinic to self-collect blood from the upper arm. We compared HIV antigen/antibody, quantitative RPR and creatinine assay results using paired Tasso and venipuncture serum specimens.

Most clients were cisgender men (42/46, 91\%) and currently using PrEP (33/37, 89\%). Of 46 participants, 40 (87\%) collected a blood sample sufficient to complete $\geq 1$ test. Percent positive agreement and negative percent agreement (PPA and NPA, respectively) for $34 \mathrm{HIV}$ specimens was 100\% (7/7 HIV positive). Of 30 paired qualitative RPR specimens, PPA was $100 \%$ (10/10 samples) and NPA was 95\% (19/20 samples). Differences between 9 paired quantitative RPR titers were all $\leq 1$ dilution. Paired creatinine results $(n=9)$ were strongly and positively correlated $\left(\mathrm{r}^{2}=0.84, \mathrm{p}=0.008\right)$ with a median difference of $0.05 \mathrm{mg} / \mathrm{dL}$ (range: -0.01-0.20).

Self-collection of blood specimens adequate for recommended PrEP laboratory testing is feasible and results show high levels of agreement with venipuncture samples. The Tasso device shows promise for use in mailed sampling kits for HBPM.

\section{P116 FACTORS ASSOCIATED WITH EXCHANGE SEX AMONG WOMEN AND MEN WHO INJECT DRUGS - 23 US CITIES, 2018}

J Rushmore*, K Buchacz, D Broz, C Agnew-Brune, M Johnson Jones, S Cha. Centers for Disease Control and Prevention, Atlanta, USA

\subsection{6/sextrans-2021-sti.237}

Background People who inject drugs (PWID) and exchange sex face disproportionate burdens of HIV and sexually transmitted infections (STIs). Studies examining risk factors for exchange sex are limited among PWID, especially men, and could help tailor prevention efforts.

Methods We used 2018 National HIV Behavioral Surveillance data to assess prevalence of exchange sex among PWID, specifically women and men with a history of sex with men (MSM). Exchange sex included receiving money/drugs for sex from $\geq 1$ male partner(s) during the past year. We conducted bivariate analyses that accounted for each respondent's city and network size to examine associations between exchange sex and sociodemographic characteristics, risk behaviors, and HIV/STI diagnoses.

Results Among 4,657 PWID (3,391 women; 1,266 MSM), over one-third reported exchange sex (women: 36.2\%; MSM: $34.8 \%$ ). Women who exchanged sex (WES) tested HIV-positive more frequently $(8.0 \%$ vs. $4.8 \%, \mathrm{P}=0.005)$ and reported more frequent recent bacterial STIs $(13.4 \%$ vs. $5.1 \%$, $\mathrm{P}<0.001)$ than other women. Men who exchanged sex with men (MESM) showed similar trends (HIV: $17.5 \%$ vs. $14.6 \%$, $\mathrm{P}=0.181$; STIs: $12.7 \%$ vs. $6.1 \%, \mathrm{P}<0.001)$. WES and MESM were more likely to be uninsured (women: $45.7 \%$ vs. $33.3 \%$, $\mathrm{P}<0.001$; MSM: $43.3 \%$ vs. $31.6 \%, \mathrm{P}=0.002)$ and recently homeless (women: $41.8 .4 \%$ vs. $25.2 \%, \quad \mathrm{P}<0.001$; MSM: $39.6 \%$ vs. $17.2 \%, \mathrm{P}<0.001)$. WES also reported higher frequencies of unemployment $(37.2 \%$ vs. $29.2 \%, \mathrm{P}=0.001)$ and incarceration $(42.5 \%$ vs. $33.4 \%, \mathrm{P}<0.001)$ than other women. WES and MESM were more likely to engage in polydrug use and risky sexual behaviors (e.g. number of condomless anal sex partners).

Conclusion Men are often overlooked in interventions for PWID who exchange sex; however, we found women and MSM had similar frequencies of and risk factors for exchange sex among PWID. Interventions for PWID should be available for women and men who report exchange sex, while integrating opportunities for HIV/STI testing and preventative care. 